\title{
AN INTERNAL AND AN EXTERNAL CHARACTERIZATION OF CONVERGENCE SPACES IN WHICH ADHERENCES OF FILTERS ARE CLOSED
}

\author{
EVA LOWEN-COLEBUNDERS
}

\begin{abstract}
The purpose of this note is to give necessary and sufficient conditions for a convergence space $(X, q)$ such that for every filter on $X$ its adherence is a closed subset of $(X, q)$. An internal characterization of this property is given by weakening the diagonal condition of Kowalsky. An external characterization is given using a hyperspace structure on the collection of all closed subsets of the given space. It will be shown that a convergence space has closed adherences if and only if the hyperspace is compact.
\end{abstract}

Introduction. We shall investigate what conditions a convergence space has to fullfill in order that every filter has a closed adherence. In general, adherences of filters in a convergence space are not closed. This is one of the essential differences between a topological space and a general convergence space. Nevertheless many nontopological convergence spaces are known in which adherences of filters are closed (for example, almost topological spaces and diagonal convergence spaces).

We shall give two characterizations of spaces with closed adherences. An internal characterization will be obtained by weakening the diagonal condition of Kowalsky. We shall introduce weakly diagonal convergence spaces and show that these are exactly the convergence spaces with closed adherences. For pretopological spaces the notions almost topological, diagonal and weak diagonal are all equivalent with the idempotency of the closure operator. We show that in general the class of weakly diagonal spaces is strictly larger than the class of diagonal or almost topological spaces but strictly smaller than the class of spaces with an idempotent closure operator.

An external characterization for a convergence space to have closed adherences will be given using a hyperspace structure on the collection of closed subsets of the given space. In fact the structure considered is the Choquet hyperspace described in [2, p. 87] which also corresponds to the convergence of nets of closed sets as defined by Frolík in [4, p. 169] and by Mrówka in $[9$, p. 59]. The adherences of filters on a given convergence space are closed if and only if the hyperspace is compact.

For all notational conventions and for definitions on convergence spaces

Received by the editors December 30, 1977.

AMS (MOS) subject classifications (1970). Primary 54A05, 54A20, 54B20.

Key words and phrases. Convergence spaces, closed adherences, almost topological spaces, diagonal spaces, hyperspace. 
we refer to [1], [2], [3] or [5]. We recall those being used frequently. A convergence space $(X, q)$ is a set $X$ together with a map $q$ which to any point $x$ of $X$ assigns a family $q x$ of filters on $X$. The filters in $q x$ are said to $q$-converge to $x$. For every $x \in X, q x$ contains $\dot{x}$, the filter generated by $\{x\}$. If $\mathscr{F}$ belongs to $q x$ then so do $\mathscr{F} \cap \dot{x}$ and every filter which is finer than $\mathscr{F}$.

Sometimes stronger axioms are imposed.

A convergence space is pseudotopological if a filter $\mathscr{F}$ converges to some point $x$ if and only if all ultrafilters finer than $\mathscr{F}$ converge to $x$. A convergence space is pretopological if for every point $x$ the intersection of all filters converging to $x$ converges to $x$. With a convergence space $(X, q)$ one associates the pseudotopological, the pretopological and the topological modification which are respectively the finest pseudotopological, pretopological and topological structure on $X$ coarser than $q$ and which are denoted by $\pi(q), \psi q$ and $\lambda q$ respectively.

A closure operator on a convergence space is defined in the following way. If $A$ is a subset of $X$ and $x \in X$ then $x \in \bar{A}$ if there exists a filter $\mathscr{F}$ containing $A$ and converging to $x . A$ is defined to be open if every filter converging to some point of $A$ contains $A$. $A$ is closed if its complement is open which is equivalent to $A=\bar{A}$. If $\mathcal{F}$ is a filter on $X$ then its adherence $\alpha_{q} \mathscr{F}$ is the set of points $x$ of $X$ for which there exists a filter finer than $\mathscr{F}$ and converging to $x$. If $X$ is a set then the collection of all filters on $X$ is denoted by $\mathbf{F}(X)$.

1. An internal characterization of convergence spaces with closed adherences.

DEFINITION 1.1. A convergence space $(X, q)$ is said to have closed adherences if for every filter $\mathscr{F}$ on $X$ the adherence $\alpha_{q} \mathscr{F}$ is a closed subset of $(X, q)$.

From this definition it is clear that almost topological convergence spaces (i.e. spaces for which the pseudotopological modification is topological) [6, p. 485] have closed adherences. If $\mathcal{F}$ is a filter on an almost topological space $(X, q)$ then we have $\alpha_{q} \mathscr{F}=\alpha_{\pi q} \mathscr{F}=\alpha_{\lambda q} \mathscr{F}$.

It is clear that spaces with closed adherences have a closure operator which is idempotent since the closure of a subset $A$ is the adherence of the filter [ $A]$ generated by $A$.

DEFINITION 1.2. A convergence space $(X, q)$ is weakly diagonal if for every $x \in X$, for every filter $\mathcal{F}$ converging to $x$, for every mapping $\eta$ from $X$ into $\mathbf{F}(X)$ such that $\eta$ converges to $y$ for every $y \in X$ and for every $F \in \mathcal{F}$ we have that $x$ is an adherence point of the filter $\mathcal{G}(\eta, F)$ where $\mathcal{G}(\eta, F)=$ $\bigcap_{y \in F} \eta y$.

It is clear that in the previous definition it suffices to consider ultrafilters $\mathscr{F}$ and mappings $\eta$ such that $\eta y$ is an ultrafilter for every $y \in X$. It is also clear that instead of checking the condition for every $F \in \mathscr{F}$ it is sufficient to consider all $F$ in a base of $\mathscr{F}$.

THEOREM 1.3. A convergence space has closed adherences if and only if it is weakly diagonal. 
Proof. Suppose $(X, q)$ has closed adherences. Let $x \in X, \mathscr{F}$ a filter on $X$ $q$-converging to $x$ and $\eta: X \rightarrow \mathbf{F}(X)$ a map such that $\eta$ converges to $y$ for every $y \in X$. For $F \in \mathscr{F}$ we have $F \subset \alpha_{q} \mathcal{G}(\eta, F)$ since $y \in F$ implies $\eta y \supset$ $\mathcal{G}(\eta, F)$ and thus $y \in \alpha_{q} \mathcal{G}(\eta, F)$. It follows that $\alpha_{q} \mathcal{G}(\eta, F) \in \mathscr{F}$. Since $\mathscr{F}$ converges to $x$ we have $x \in \overline{\alpha_{q} \mathcal{G}(\eta, F)}=\alpha_{q} \mathcal{G}(\eta, F)$. Suppose $(X, q)$ is weakly diagonal. Let $\mathcal{F}$ be a filter on $X$. If $x \in \frac{q}{\alpha_{q} \mathscr{F}}$ then take a filter $\mathcal{H}$ converging to $x$ and containing $\alpha_{q} \mathscr{F}$. For $y \in \alpha_{q} \mathscr{F}$ let $\mathcal{F F}_{y}$ be a filter converging to $y$ and finer than $\mathscr{F}$. Consider $\eta: X \rightarrow \mathbf{F}(X), \eta y=\dot{y}$ if $y \notin \alpha_{q} \mathscr{F}$ and $\eta y=\mathcal{H}_{y}$ if $y \in \alpha_{q} \mathscr{F}$. Then we have $\mathscr{F} \subset \mathcal{G}\left(\eta, \alpha_{q} \mathscr{F}\right)$ and therefore $x \in \alpha_{q} \mathscr{F}$.

As defined by Kowalsky a convergence space is diagonal if for every $x, \mathcal{F}$ and $\eta$ satisfying the conditions of 1.2 the filter $\kappa \eta \mathscr{F}=\sup _{F \in \mathscr{F}} \mathcal{G}(\eta, F)$ converges to $x$. For a more detailed definition we refer to [7, p. 312]. It is clear that diagonal convergence spaces are weakly diagonal and from the previous theorem it follows that diagonal spaces have closed adherences. This result is slightly stronger than the statement in [7, p. 312] which says that diagonal convergence spaces have a closure operator which is idempotent. For pretopological spaces all the notions we are dealing with coincide as follows from the next theorem.

THEOREM 1.4. For a pretopological space the following properties are equivalent:

(1) almost topological,

(2) diagonal,

(3) weakly diagonal,

(4) idempotent closure operator.

Proof. $(1) \Rightarrow(2)$ is the only nontrivial implication. It follows from the fact that a topological space is diagonal as was shown in [7, p. 312].

Examples can be given to show that in the case of a general convergence space the only existing implications between the four properties of the previous theorem are those being pointed out in the previous sections. It can be shown that an almost topological space need not be diagonal and that a diagonal space need not be almost topological. Here we shall only construct examples to show that a space with an idempotent closure operator does not necessarily have closed adherences and that a space having closed adherences need not be diagonal or almost topological.

EXAMPLE 1.5. Take the set $\mathbf{N}$ of the natural numbers and choose a nonprincipal ultrafilter $\mathcal{Q}$ on $\mathbf{N}$. We define $q$ to be the following pseudotopological structure on $\mathbf{N}$. An ultrafilter $Q \int$ converges to $x$, where $x \neq 0$, if and only if $\mathscr{W}=\dot{x}$ or $W$ is a nonprincipal ultrafilter. An ultrafilter $W$ converges to 0 if and only if $\mathscr{W}=\dot{0}$ or $\mathscr{W}$ is a nonprincipal ultrafilter different from $\mathcal{Q}$. Then $(\mathbf{N}, q)$ has an idempotent closure operator since $\psi q$ is the topology of finite complements on $\mathbf{N}$. But $\alpha_{q} \mathcal{Q}=\mathbf{N} \backslash\{0\}$ which is not $q$-closed. 
Example 1.6. Let $X=\left\{x_{0}, x_{1}, \ldots\right\}$ and $X^{\prime}=\left\{x_{0}^{\prime}, x_{1}^{\prime}, \ldots\right\}$ be two disjoint copies of $\mathbf{N}$. If $A \subset X$ then the copy of $A$ in $X^{\prime}$ is denoted by $A^{\prime}$. If ข is a filter on $X$ then $\mho^{\prime}$ is its copy on $X^{\prime}$. Choose a nonprincipal ultrafilter $\mathcal{U}$ on $X$. For every $U \in \mathcal{U}$ choose a nonprincipal ultrafilter $\mathscr{W}_{U}$ different from $\mathcal{Q}$ and containing $U$. On $Y=X \cup X^{\prime}$ we define a pseudotopological structure $q$ as follows. In $x_{0}$ the convergent ultrafilters are $\dot{x}_{0},[Q]$ and every [ $\mathcal{W}_{U}^{\prime}$ ] for $U \in \mathcal{Q}$. For every $n \neq 0$ the convergent ultrafilters in $x_{n}$ are $\dot{x}_{n}$ and $\dot{x}_{n}^{\prime}$. For every $n \in \mathbf{N}$ the only convergent ultrafilter in $x_{n}^{\prime}$ is $\dot{x}_{n}^{\prime}$.

The space $(Y, q)$ is not (almost) topological because $\cap_{U \in \mathcal{U}}\left[\mathcal{W}_{U}^{\prime}\right] \subset\left[\mathcal{Q}^{\prime}\right]$ implies that $\cap_{U \in \mathcal{Q}}\left[\tilde{D}_{U}^{\prime}\right]$ does not converge to $x_{0} ;(Y, q)$ is not diagonal. Consider the filter [थ] converging to $x_{0}$ and the map $\eta: Y \rightarrow \mathbf{F}(Y)$ with $\eta x_{n}=\dot{x}_{n}^{\prime}$ for all $n \neq 0, \eta x_{0}=\dot{x}_{0}$ and $\eta x_{n}^{\prime}=\dot{x}_{n}^{\prime}$ for all $n \in \mathbf{N}$. Then we have $\kappa \eta[\mathcal{U}]=\sup _{A \in[थ]} \mathcal{G}(\eta, A)=\sup _{U \in \mathcal{U}} \mathcal{G}\left(\eta, U \backslash\left\{x_{0}\right\}\right)=\left[\mathcal{Q} \mathrm{U}^{\prime}\right]$ which does not converge to $x_{0}$.

But $(Y, q)$ has closed adherences. In fact we show that it is weakly diagonal. For principal ultrafilters $\dot{x}_{n}$ converging to $x_{n}$ or $\dot{x}_{n}^{\prime}$ converging to $x_{n}^{\prime}$ the control for weak diagonality is trivial. Next consider the case of a principal filter $\dot{x}_{n}^{\prime}$ converging to $x_{n}$. Clearly for every $\eta$ we have $\mathcal{G}(\eta$, $\left.\left\{x_{n}^{\prime}\right\}\right)=\dot{x}_{n}^{\prime}$ which converges to $x_{n}^{\prime}$.

Thirdly we deal with the case of the ultrafilter [थ] converging to $x_{0}$. Let $\eta$ : $Y \rightarrow \mathbf{F}(Y)$ be such tha $\eta y \in q y$ for every $y \in Y$. Let $U \in \mathcal{U}$. If $x_{0} \in U$ then clearly $\mathcal{G}(\eta, U) \subset \eta x_{0}$ whence $x_{0} \in \alpha_{q} \mathcal{G}(\eta, U)$. If $x_{0} \notin U$ then consider $A=\{x \in U \mid \eta x=\dot{x}\}$ and $B=\left\{x \in U \mid \eta x=\dot{x}^{\prime}\right\}$. Since $A \cup B=U$ we have either $A \in \mathcal{U}$ or $B \in \mathcal{Q}$. If $A \in \mathcal{U}$ then $\mathcal{G}(\eta, U) \subset \mathcal{G}(\eta, A)=[A] \subset$ [थ] and thus $x_{0} \in \alpha_{q} \mathcal{G}(\eta, U)$. If $B \in \mathcal{U}$ then $\mathcal{G}(\eta, U) \subset \mathcal{G}(\eta, B)=\left[B^{\prime}\right] \subset$ ข $\int_{B}^{\prime}$ and thus $x_{0} \in \alpha_{q} \mathcal{G}(\eta, U)$. Finally we consider the case of an ultrafilter [थ $\mathcal{W}_{U}^{\prime}$ ] converging to $x_{0}$ for some $U \in \mathcal{Q}$. Let $\eta: Y \rightarrow \mathbf{F}(Y)$ be such that $\eta y \in q y$ for every $y \in Y$. Let $Z^{\prime} \in \mathcal{W}_{U}^{\prime}$. Then $\mathcal{G}\left(\eta, Z^{\prime}\right)=\left[Z^{\prime}\right] \subset\left[\mathcal{W}_{U}^{\prime}\right]$ and thus $x_{0} \in \alpha_{q} \mathcal{G}\left(\eta, Z^{\prime}\right)$.

REMARK 1.7. Several other conclusions follow from the previous example. The family of all convergence spaces with closed adherences on a fixed set is not a sublattice of the complete lattice of all convergence structures on that set. Take for instance the space $(Y, q)$ described in the previous example. On the other hand consider the following topology $p$ on $Y$ defined by its neighborhoodfilters $\mathscr{V}\left(x_{0}\right)=\dot{x}_{0} \cap[\mathcal{U}] \cap\left[\mathcal{U}^{\prime}\right], \widetilde{V}\left(x_{n}\right)=\dot{x}_{n} \cap \dot{x}_{n}^{\prime}$ if $n \neq 0$ and $\mathcal{V}\left(x_{n}^{\prime}\right)=\dot{x}_{n}^{\prime}$ for all $n \in \mathbf{N}$. Then $q \vee p$ is not weakly diagonal.

A similar example can be made to show that the infimum of two topological spaces need not be weakly diagonal.

Some positive results in this context are the following. If $f: X \rightarrow(Y, p)$ is a map and $(Y, p)$ has closed adherences then the initial structure on $X$ has closed adherences. In particular any subspace of a space with closed adherences has closed adherences. The convergence sum of any family of spaces with closed adherences has closed adherences. Products and quotients however fail to preserve the property of having closed adherences. 
2. An external characterization of convergence spaces with closed adherences. Let $(X, q)$ be a convergence space and $\mathscr{P}(X)$ the collection of all closed subsets of $(X, q)$. On $\mathscr{P}(X)$ a natural hyperspace structure $c(q)$ can be defined as will be done in 2.1 .

We first need some preliminary notations. If $\Psi$ is a filter on $\mathscr{P}(X)$ and $\mathbb{Q} \in \Psi$ then we put $E_{\mathscr{Q}}=\cup\{A \mid A \in \mathbb{Q}\}$. If $\Psi \neq \dot{\varnothing}$ then $\left\{E_{\mathscr{Q}} \mid \mathbb{Q} \in \Psi\right\}$ is a filterbase on $X$ generating a filter which is denoted by $\mathscr{F}(\Psi)$. The adherence of $\mathscr{F}(\Psi)$ in $(X, q)$ will be called the supremum of $\Psi$ and is denoted by sup $\Psi$. If $\Psi=\dot{\varnothing}$ then we define sup $\Psi=\varnothing$. If $\mathscr{F}$ is a filter on $X$ and if $F \in \mathcal{F}$ then we put $\bigodot_{F}=\{E \in \mathcal{P}(X) \mid E \cap F \neq \varnothing\}$. The collection $\left\{\bigcup_{F} \mid F \in \mathcal{F}\right\}$ is a filterbase on $\mathcal{P}(X)$ generating a filter $\Lambda(\mathscr{F})$. We define the infimum of $\Psi$, denoted by inf $\Psi$, to be the set of points $x$ of $X$ for which there exists a filter $\mathscr{F}$ on $X q$-converging to $x$ with $\Lambda(\mathscr{F}) \subset \Psi$.

Definition 2.1. A filter $\Psi$ on $\mathscr{P}(X) c(q)$-converges to some $E \in \mathscr{P}(X)$ if and only if $\sup \Psi=\inf \Psi=E$.

It is easily checked that $c(q)$ is a convergence structure on $\mathscr{P}(X)$. If $(X, q)$ is a topological space with neighborhoodfilter $\mathscr{V}(x)$ in every $x \in X$ then the definitions of sup $\Psi$ and inf $\Psi$ reduce to the following.

Let $x \in X . x \in \sup \Psi$ if and only if for every $V \in \mathcal{V}(x)$ and for every $\mathbb{Q} \in \Psi$ there exists an $A \in \mathbb{Q}$ such that $V \cap A \neq \varnothing . x \in$ inf $\Psi$ if and only if for every $V \in \mathcal{V}(x)$ there exists an $Q \in \Psi$ such that $V \cap A \neq \varnothing$ for every $A \in \mathbb{Q}$.

Hence it is clear that for a topological space $(X, q)$ the filter convergence on $(\mathscr{P}(X), c(q))$ corresponds to the convergence of nets in $\mathscr{P}(X)$ described by Frolík [4, p. 169] and Mrówka [9, p. 59]. On $2^{X}$, the collection of nonempty closed subsets of a convergence space, $c(q)$ induces a structure which has been described in [8] and which coincides with the hyperspace structure of Choquet in case $(X, q)$ is topological [2, p. 87].

THEOREM 2.2. A convergence space $(X, q)$ has closed adherences if and only if $(\mathscr{P}(X), c(q))$ is compact.

Proof. Suppose $(X, q)$ has closed adherences and let $\Omega$ be an ultrafilter on $\mathscr{P}(X)$. Either $\Omega=\dot{\varnothing}$ and then $\Omega c(q)$-converges to $\varnothing$ or $\Omega \neq \dot{\varnothing}$. Then sup $\Omega=\alpha_{q} \mathscr{F}(\Omega)$ which belongs to $\mathscr{P}(X)$. It is easily checked that for every filter $\mathscr{F}$ on $X$ we have $\Lambda(\mathscr{F}) \subset \Omega$ if and only if $\mathscr{F} \vee \mathscr{F}(\Omega)$ exists. It follows that inf $\Omega=\sup \Omega$. Hence $\Omega c(q)$-converges to $\alpha_{q} \mathscr{F}(\Omega)$.

For the converse, suppose $(\mathscr{P}(X), c(q))$ is compact and let $\mathscr{F}$ be a filter on $X$. Let $\Gamma(\mathscr{F})$ be the filter on $\mathscr{P}(X)$ generated by the base $\left\{Q_{F} \mid F \in \mathscr{F}\right\}$ where $\mathbb{Q}_{F}=\{E \in \mathscr{P}(X) \mid E \subset F\}$. Then there exists an ultrafilter $\Omega$ on $\mathscr{P}(X)$ such that $\Omega \supset \Gamma(\mathscr{F})$ and $\mathscr{F}(\Omega) \subset \mathcal{F}$. Suppose on the contrary that for every ultrafilter $\Omega$ finer than $\Gamma(\mathscr{F})$ we have an $Q_{\Omega} \in \Omega$ such that $E_{\mathcal{Q}_{\Omega}} \notin \mathcal{F}$. Then we could find a finite number of these ultrafilters say $\Omega_{1} \cdots \Omega_{n}$ such that $\cup{ }_{i=1}^{n} \mathbb{Q}_{\Omega_{i}} \in \Gamma(\mathscr{F})$ (cf. Lemma 1.1 of [8]). Let $F \in \mathscr{F}$ such that $Q_{F} \subset$ $\cup_{i=1}^{n} \mathbb{Q}_{\Omega_{i}}$. For $i \in\{1, \ldots, n\}$ we have $E_{\mathbb{Q}_{\Omega i}} \not F$ so we could choose $x_{i} \in F$ 
with $x_{i} \notin E_{\mathbb{Q}_{R i}}$. Then $\left\{x_{1}, \ldots, x_{n}\right\} \in \mathbb{Q}_{F}$. This would imply that for some $j \in\{1, \ldots, n\}$ the set $\left\{x_{1}, \ldots, x_{n}\right\} \in \mathbb{Q}_{\Omega_{j}}$. But then $x_{j} \in E_{\mathbb{Q}_{R j}}$ which is a contradiction.

Now let $\Omega$ be an ultrafilter on $\mathscr{P}(X)$ such that $\Omega \supset \Gamma(\mathscr{F})$ and $\mathscr{F}(\Omega) \subset \mathscr{F}$. Since $\mathscr{F}(\Omega) \supset \mathscr{F}$ if and only if $\Omega \supset \Gamma(\mathscr{F})$ it follows that $\mathscr{F}(\Omega)=\mathscr{F}$. Since $\Omega$ $c(q)$-converges it follows that $\alpha_{q} \mathscr{F}=\alpha_{q} \mathscr{F}(\Omega)=\sup \Omega \in \mathscr{P}(X)$.

\section{REFERENCES}

1. N. Bourbaki, Topologie générale, 4th ed., Éléments de mathématique, Fasc. II, Chap. 1, Hermann, Paris, 1965.

2. G. Choquet, Convergences, Ann. Univ. Grenoble Sect. Sci. Math. Phys. 23 (1948), 57-112.

3. H. Fisher, Limesraüme, Math. Ann. 137 (1959), 269-303.

4. Z. Frolik, Concerning topological convergence of sets, Czechoslovak Math. J. 10 (1960), 168-180.

5. D. Kent, Convergence functions and their related topologies, Fund. Math. 54 (1964), 125-133.

6. D. Kent and G. Richardson, Regular completions of Cauchy spaces, Pacific J. Math. 51 (1974), 483-490.

7. H. Kowalsky, Limesraüme und Komplettierung, Math. Nachr. 12 (1954), 301-340.

8. E. Lowen-Colebunders, The Choquet hyperspace structure for a convergence space, (to appear).

9. S. Mrówka, Some comments on the space of subsets, Lecture Notes in Math. 171 (1970), $59-63$.

aAngesteld Navorser N. F. W. O., Departement voor Wiskunde, Vrije Universiteit Brussel, Pleinlaan 2, 1050 Brussel, België 